PROCEEDINGS OF THE

AMERICAN MATHEMATICAL SOCIETY

Volume 139, Number 12, December 2011, Pages 4487-4496

S 0002-9939(2011)10849-9

Article electronically published on April 21, 2011

\title{
NON-ISOMORPHISM INVARIANT BOREL QUANTIFIERS
}

\author{
FREDRIK ENGSTRÖM AND PHILIPP SCHLICHT
}

(Communicated by Julia Knight)

\begin{abstract}
Every isomorphism invariant Borel subset of the space of structures on the natural numbers in a countable relational language is definable in $\mathscr{L}_{\omega_{1} \omega}$ by a theorem of Lopez-Escobar. We derive variants of this result for stabilizer subgroups of the symmetric group $\operatorname{Sym}(\mathbb{N})$ for families of relations and non-isomorphism invariant generalized quantifiers on the natural numbers such as "for all even numbers". Moreover we produce a binary quantifier $Q$ for every closed subgroup of $\operatorname{Sym}(\mathbb{N})$ such that the Borel sets of structures invariant under the subgroup action are exactly the sets of structures definable in $\mathscr{L}_{\omega_{1} \omega}(Q)$.
\end{abstract}

\section{INTRODUCTION}

It is useful to represent structures with universe the natural numbers in a given countable relational signature $\tau$ by elements of the associated logic space

$$
X_{\tau}=\prod_{R \in \tau} 2^{\mathbb{N}^{a(R)}},
$$

where $X_{\tau}$ is equipped with the product topology and $a$ denotes arity. For example, the linear orders form a closed subset of the logic space for a single binary relation. The isomorphism classes of structures with universe $\mathbb{N}$ are orbits of the symmetric group $\operatorname{Sym}(\mathbb{N})$ under permutation. This connection between countable model theory and descriptive set theory is important for investigating isomorphism relations for classes of countable structures.

The relationship between invariant Borel classes of structures on the natural numbers and theories is described by the Lopez-Escobar theorem [3. Theorem 16.8], which states that every invariant Borel subset of the logic space is defined by a sentence in $\mathscr{L}_{\omega_{1} \omega}$. This is an easy consequence, due to Scott, of the interpolation theorem for $\mathscr{L}_{\omega_{1} \omega}$ 4: If $\varphi$ is an $\mathscr{L}_{\omega_{1} \omega}$-sentence in the signature $\sigma$ and $\psi$ is an $\mathscr{L}_{\omega_{1} \omega}$-sentence in the signature $\tau$ such that $\varphi \rightarrow \psi$ holds in all countable models, then there is an $\mathscr{L}_{\omega_{1} \omega}$-interpolant $\theta$ in the signature $\sigma \cap \tau$ such that $\varphi \rightarrow \theta$ and $\theta \rightarrow \psi$

Received by the editors March 19, 2010 and, in revised form, October 19, 2010

2010 Mathematics Subject Classification. Primary 03E15, 03C80.

Part of the work in this paper was done while visiting the Institut Mittag-Leffler. The authors would like to thank the Institut Mittag-Leffler for support and also the anonymous referee for several valuable comments and suggestions.

The first author was partially supported by the EUROCORE LogICCC LINT program and the Swedish Research Council.

The second author received support from the European Science Foundation for the activity 'New Frontiers of Infinity: Mathematical, Philosophical and Computational Prospects'.

(C) 2011 American Mathematical Society Reverts to public domain 28 years from publication 
hold in all countable models. To derive the Lopez-Escobar theorem from interpolation, note that every Borel set is defined by an $\mathscr{L}_{\omega_{1} \omega}$-sentence from a sequence of parameters $n_{i} \in \mathbb{N}$. If we use constants $c_{i}$ and $d_{i}$ for each $n_{i}$, the assumption that the Borel set is isomorphism invariant implies that there is an $\mathscr{L}_{\omega_{1} \omega}$-interpolant (without parameters) defining the set. Vaught [5] found a different proof which has the advantage that it generalizes to the logic space for structures of higher cardinalities.

In this paper we consider classes of structures on the natural numbers which are invariant under the action of a closed subgroup of $\operatorname{Sym}(\mathbb{N})$ instead of isomorphism. Vaught's proof is generalized to this setting in section 2. A natural example is the class of models with domain $\mathbb{N}$ of a first-order theory in which a given $k$-ary relation has a fixed interpretation as a subset of $\mathbb{N}^{k}$. The set of permutations which fix this relation forms a closed subgroup of $\operatorname{Sym}(\mathbb{N})$, and the class is invariant under the subgroup action.

We then focus on Lindström quantifiers (generalized quantifiers) on the natural numbers without the requirement of invariance under isomorphism; i.e., a quantifier $Q$ of type $\langle k\rangle$ is a set of $k$-ary relations and $\operatorname{Aut}(Q)$ is the group of those permutations which fix $Q$ as a set. The logic $\mathscr{L}_{\omega_{1} \omega}(Q)$ is defined as $\mathscr{L}_{\omega_{1} \omega}$ augmented by the quantifier $Q$, where $\mathbb{N} \vDash Q x \varphi(x)$ is interpreted as $\left\{a \in \mathbb{N}^{k}: \mathbb{N} \vDash \varphi(a)\right\} \in Q$; the process of checking whether an $\mathscr{L}_{\omega_{1} \omega}(Q)$-sentence holds in a structure can be thought of as running an algorithm along $L_{\omega_{1}^{C K(x)}}[x]$ for some $x \in 2^{\omega}$ with input the atomic diagram which feeds in external information about $Q$. A version of the Lopez-Escobar theorem is proved for a class of monotone upwards quantifiers such as $\forall, \exists$, "for some prime number", or "for all even numbers or for all odd numbers". Moreover for every closed subgroup of $\operatorname{Sym}(\mathbb{N})$ a binary quantifier $Q$ is produced such that the Borel sets of structures invariant under the subgroup action are exactly the sets definable in $\mathscr{L}_{\omega_{1} \omega}(Q)$.

Other types of quantifiers for which a version of the Lopez-Escobar theorem can be proved are clopen quantifiers and finite boolean combinations of principal quantifiers; this is presented in section 3 . In section 4 , we observe that some of the results generalize to the logic space for structures of size $\kappa$ for uncountable cardinals $\kappa$ with $\kappa^{<\kappa}=\kappa$.

\section{VARIANTS OF THE LOPEZ-ESCOBAR THEOREM}

Let $\mathcal{F}$ denote a countable family of relations on the natural numbers. Every relation in $\mathcal{F}$ has a symbol representing it in $\mathscr{L}_{\omega_{1} \omega}(\mathcal{F})$. The following two kinds of definability are relevant.

Definition 1. A set $A \subseteq \mathbb{N}^{k}$ is definable in $\mathscr{L}_{\omega_{1} \omega}(\mathcal{F})$ if there is an $\mathscr{L}_{\omega_{1} \omega}(\mathcal{F})$ sentence $\varphi$ such that $n \in A$ if and only if $\mathbb{N} \vDash \varphi(n)$.

Definition 2. Suppose $\tau$ is a countable relational signature disjoint from the symbols for $\mathcal{F}$. A set $A \subseteq X_{\tau}$ is definable in $\mathscr{L}_{\omega_{1} \omega}(\mathcal{F})$ if there is an $\mathscr{L}_{\omega_{1} \omega}(\mathcal{F})$-sentence $\varphi$ such that for all $M \in X_{\tau}, M \in A$ if and only if $\langle M, \mathcal{F}\rangle \vDash \varphi$.

The second definition covers subsets of the space $2^{\mathbb{N}^{k}}$, since this is the logic space for a single $k$-ary relation. We will freely identify subsets of $\mathbb{N}^{k}$ with their characteristic functions. 
2.1. Variants relative to relations. We present variants of the Lopez-Escobar theorem for closed subgroups of $\operatorname{Sym}(\mathbb{N})$ and $\mathscr{L}_{\omega_{1} \omega}(\mathcal{F})$. Let $G \leq H$ mean that $G$ is a subgroup of $H$.

Definition 3. Suppose $G \leq \operatorname{Sym}(\mathbb{N})$. The $G$-orbits of a tuple $a \in \mathbb{N}<\omega$ and a set $A \subseteq \mathbb{N}^{<\omega}$ are defined $\operatorname{as}_{\operatorname{Orb}_{G}}(a)=\{g(a): g \in G\}$ and $\operatorname{Orb}_{G}(A)=\{g(a): g \in$ $G, a \in A\}$.

When $G$ is understood from the context, we write $\operatorname{Orb}(a)$ and $\operatorname{Orb}(A)$ for the $G$-orbits. Recall the standard

Fact 4. The closed subgroups of $\operatorname{Sym}(\mathbb{N})$ are exactly the automorphism groups of countable relational structures on $\mathbb{N}$. In fact, if $\mathcal{F}$ is the family of orbits of a closed group $G \leq \operatorname{Sym}(\mathbb{N})$, then $G=\operatorname{Aut}(\mathcal{F})$.

See for example [2, Theorem 2.4.4] for a proof.

Proposition 5. Suppose $G \leq \operatorname{Sym}(\mathbb{N})$ is closed and $\mathcal{F}$ is the family of $G$-orbits of tuples. Suppose the signature $\tau$ is disjoint from the symbols for $\mathcal{F}$. Then a subset of $X_{\tau}$ is Borel and $G$-invariant if and only if it is definable in $\mathscr{L}_{\omega_{1} \omega}(\mathcal{F})$.

Proof. The proof of the forward implication is analogous to Vaught's proof [5]. Following the proof of [3, Theorem 16.8], we replace the set of injections $k \rightarrow \mathbb{N}$ with the orbit of $\langle 0,1, \ldots, k-1\rangle$. Note that the Baire category theorem holds for $G$ since $G$ is closed in $\operatorname{Sym}(\mathbb{N})$. For every Borel set $A \subseteq X_{\tau}$, there is a sequence of $\mathscr{L}_{\omega_{1} \omega}(\mathcal{F})$-formulas $\varphi_{k}$ such that for all $M \in X_{\tau}$ and $a \in \mathbb{N}^{k}, M \models \varphi_{k}(a)$ if and only if $g(M) \in A$ for comeager many $g \in G$ with $a \subseteq g^{-1}$. This is proved by induction on the Borel rank. The argument for the other implication is a straightforward induction on formulas of $\mathscr{L}_{\omega_{1} \omega}(\mathcal{F})$.

While every $G_{\delta}$ subgroup of $\operatorname{Sym}(\mathbb{N})$ is closed [1, Proposition 1.2.1], the proposition is false for some $F_{\sigma}$ subgroups. We write $A={ }^{*} B$ if $A \triangle B$ is finite and $A \subseteq \subseteq^{*} B$ if $A-B$ is finite. Suppose $A \subseteq \mathbb{N}$ is infinite and co-infinite and let $G=\left\{g \in S_{\infty}: g(A)={ }^{*} A\right\}$. Then $G$ is $F_{\sigma}$ and has the same orbits as $\operatorname{Sym}(\mathbb{N})$. However, the set $\left\{X: A \subseteq^{*} X\right\}$ is $G$-invariant, but not $\operatorname{Sym}(\mathbb{N})$-invariant and hence not definable from the orbits of $G$.

It is natural to ask whether the family of orbits can be replaced by the orbit equivalence relation $E_{G}=\left\{\langle a, b\rangle \in \mathbb{N}^{<\omega} \times \mathbb{N}^{<\omega}: \exists g \in G(g(a)=b)\right\}$. However, $E_{G}$ may contain much less information. For example, $E_{\left\{i d_{\mathbb{N}}\right\}}$ is definable in $\mathscr{L}_{\omega_{1} \omega}$ and thus no orbit of $\left\{i d_{\mathbb{N}}\right\}$ is definable from $E_{\left\{i d_{\mathbb{N}}\right\}}$.

As a corollary we obtain a variant of Scott sentences for orbits of closed subgroups of $\operatorname{Sym}(\mathbb{N})$.

Corollary 6. Suppose $G \leq \operatorname{Sym}(\mathbb{N})$ is closed and $\mathcal{F}$ is the family of $G$-orbits of tuples. There is for each $M \in X_{\tau}$ an $\mathscr{L}_{\omega_{1} \omega}(\mathcal{F})$-sentence $\varphi_{M}^{G}$ such that for any $N \in X_{\tau}, N \models \varphi_{M}^{G}$ if and only if $N=g(M)$ for some $g \in G$.

Proof. The orbit $\operatorname{Orb}(M)=\{g(M): g \in G\}$ is Borel [2, Theorem 3.3.2].

When $G$ is the symmetric group we write $\varphi_{M}=\varphi_{M}^{\operatorname{Sym}(\mathbb{N})}$. While the sentence obtained in the corollary is not identical with the standard Scott sentence, any sentence $\varphi_{M}$ with this property is sufficient for our purpose. Here is a version of the Lopez-Escobar theorem relative to a family of relations. 
Proposition 7. Suppose $\mathcal{F}$ is a countable family of relations and that the signature $\tau$ is disjoint from the symbols for $\mathcal{F}$. Then every $\operatorname{Aut}(\mathcal{F})$-invariant Borel subset of $X_{\tau}$ is definable in $\mathscr{L}_{\omega_{1} \omega}(\mathcal{F})$.

Proof. It is sufficient to show that the $\operatorname{Aut}(\mathcal{F})$-orbits of tuples are definable in $\mathscr{L}_{\omega_{1} \omega}(\mathcal{F})$; this implies that all Aut $(\mathcal{F})$-invariant Borel sets are definable in $\mathscr{L}_{\omega_{1} \omega}(\mathcal{F})$ by Proposition 5 . Note that $\operatorname{Aut}(\mathcal{F})$ is closed. Let $M_{a}=\langle\mathbb{N}, \mathcal{F}, a\rangle$ for $a \in \mathbb{N}<\omega$. Then $a \in \operatorname{Orb}(b)$ if and only if the structures $M_{a}$ and $M_{b}$ are isomorphic if and only if $M_{b} \vDash \varphi_{M_{a}}$. Hence $\varphi_{M_{a}}$ defines the orbit of $a$.

2.2. Variants relative to quantifiers. We present variants of the Lopez-Escobar Theorem for $\mathscr{L}_{\omega_{1} \omega}(Q)$ for quantifiers $Q$. Let us suppose that $Q$ is of type $\langle k\rangle$ and $G \leq \operatorname{Sym}(\mathbb{N})$. The notions of $\mathscr{L}_{\omega_{1} \omega}(Q)$-definability for subsets of $\mathbb{N}^{l}$ and $X_{\tau}$ are analogous to those for $\mathscr{L}_{\omega_{1} \omega}(\mathcal{F})$. A permutation $f$ of $\mathbb{N}$ is said to fix $Q$ if for all $A \subseteq \mathbb{N}^{k}, A \in Q$ if and only if $f(A) \in Q$. Let $\operatorname{Aut}(Q)$ denote the group of permutations fixing $Q$. A set $A \subseteq X_{\tau}$ is called $G$-invariant if $g(A)=A$ for all $g \in G$.

Proposition 8. Suppose $Q \subseteq 2^{\mathbb{N}^{k}}$ is a Borel quantifier with closed automorphism group. Suppose the orbits of $\operatorname{Aut}(Q)$ are definable in $\mathscr{L}_{\omega_{1} \omega}(Q)$. Then a subset of $X_{\tau}$ is Borel and $\operatorname{Aut}(Q)$-invariant if and only if it is definable in $\mathscr{L}_{\omega_{1} \omega}(Q)$.

Proof. The forward implication follows from Proposition 5. We are left to show that every $\mathscr{L}_{\omega_{1} \omega}(Q)$-definable subset of $X_{\tau}$ is Borel. Note that the Borel sets are exactly the sets definable in $\mathscr{L}_{\omega_{1} \omega}$ from a sequence of natural parameters. Suppose $Q$ is defined by an $\mathscr{L}_{\omega_{1} \omega}$-sentence $\varphi_{Q}$ from a parameter sequence $s_{Q}$; i.e. $\varphi_{Q}$ mentions a sequence of constants interpreted as $s_{Q}$. We prove by induction on formulas that for every $\mathscr{L}_{\omega_{1} \omega}(Q)$-formula $\psi$ and every sequence $s$, the set of structures defined by $\psi$ from $s$ is definable by an $\mathscr{L}_{\omega_{1} \omega}$-formula from some parameter sequence and hence is Borel. Suppose $A=\left\{M \in X_{\tau}:\langle M, s\rangle \vDash Q x \chi(x)\right\}$, where $\chi$ is an $\mathscr{L}_{\omega_{1} \omega}$-formula and $s$ is a parameter sequence. Then $M \in A$ if and only if $\{a:\langle M, s\rangle \vDash \chi(a)\} \in Q$ if and only if $\left\langle\mathbb{N},\{a:\langle M, s\rangle \vDash \chi(a)\}, s_{Q}\right\rangle \vDash \varphi_{Q}$ if and only if $\left\langle M, s_{Q}, s\right\rangle \vDash \varphi_{Q}^{\chi}$, where $\varphi_{Q}^{\chi}$ is the formula obtained by replacing all occurrences of the (only) $k$-ary predicate $P(x)$ in $\varphi$ by $\chi_{Q}\left(x, c_{s}\right)$ and interpreting $c_{s}$ as $s$. Since $\varphi_{Q}^{\chi}$ is an $\mathscr{L}_{\omega_{1} \omega}$-formula, the set it defines is Borel.

An analogous result is true for countable families $\mathcal{Q}$ of quantifiers assuming $\operatorname{Aut}(\mathcal{Q})$ is closed. Note that the assumption that $\operatorname{Aut}(Q)$ is closed is essential. To see this, write $A={ }^{*} B$ if $A \triangle B$ is finite and let $Q=\left\{X: X={ }^{*} A\right\}$, where $A \subseteq \mathbb{N}$ is infinite and co-infinite. It can be easily proved by induction on $\mathscr{L}_{\omega_{1} \omega}(Q)$-formulas that $\langle\mathbb{N}, X\rangle \vDash \neg Q x \varphi(x, a)$ for all $X \neq^{*} A, \neg A$. This implies that any $\mathscr{L}_{\omega_{1} \omega}(Q)$ sentence true in a structure $\langle\mathbb{N}, X\rangle$ for some infinite and co-infinite $X \neq^{*} A, \neg A$ is true in all structures $\langle\mathbb{N}, Y\rangle$ for infinite and co-infinite $Y \neq^{*} A, \neg A$. Hence the set $\left\{X: A \subseteq^{*} X\right\}$ is $\operatorname{Aut}(Q)$-invariant but not $\mathscr{L}_{\omega_{1} \omega}(Q)$-definable.

Definition 9. Suppose $Q \subseteq 2^{\mathbb{N}^{k}}$ is monotone upwards, i.e. closed under supersets. Let us identify each natural number $n$ with the set $\{0, \ldots, n-1\}$. A function $p: n \rightarrow \mathbb{N}$ is compatible with $Q$ if and only if for every $A \subseteq n^{k}, \mathbb{N}^{k}-A \in Q$ if and only if $\mathbb{N}^{k}-p(A) \in Q$. A function $p: n \rightarrow \mathbb{N}$ is strongly compatible with $Q$ if for every $m$, there is a finite injection $q$ compatible with $Q$ extending $p$ such that $m$ is a subset of the domain and the range of $q$. 
Note that since $Q$ is monotone upwards, for all $A \subseteq n^{k}, \mathbb{N}^{k}-A \in Q$ if and only if $n^{k}-A$ extends to an element of $Q$, i.e. if and only if there is some $B \subseteq \mathbb{N}^{k}$ in $Q$ with $n^{k}-A=B \cap n^{k}$. Hence a function $p: n \rightarrow \mathbb{N}$ is compatible with $Q$ if and only if for every function $g: n^{k} \rightarrow\{0,1\}, g$ extends to (the characteristic function of) an element of $Q$ if and only if $p(g)=g \circ p^{-1}$ extends to (the characteristic function of) an element of $Q$.

Proposition 10. Suppose $Q \subseteq 2^{\mathbb{N}^{k}}$ is monotone upwards and any finite injection $p: n \rightarrow \mathbb{N}$ strongly compatible with $Q$ extends to some $f \in \operatorname{Aut}(Q)$. Then the Aut $(Q)$-orbits of tuples are definable in $\mathscr{L}_{\omega_{1} \omega}(Q)$.

Proof. A tuple $a=\left\langle a_{i}: i\langle n\rangle\right.$ is in the orbit of a tuple $b=\left\langle b_{i}: i\langle n\rangle\right.$ if and only if for every finite set $A$, there are extensions $\left\langle a_{i}: i<l\right\rangle$ of $a$ and $\left\langle b_{i}: i<l\right\rangle$ of $b$ such that the function $p$ mapping each $a_{i}$ to $b_{i}$ is well-defined and injective with $A$ a subset of its domain and range and $p$ is compatible with $Q$. The compatibility is expressible as the conjunction of $Q x \bigwedge_{t \in T} x \neq\left\langle a_{t(0)}, \ldots, a_{t(k-1)}\right\rangle \leftrightarrow Q x \bigwedge_{t \in T} x \neq$ $\left\langle b_{t(0)}, \ldots, b_{t(k-1)}\right\rangle$ over all $T \subseteq l^{k}$. This can be written as a $\mathscr{L}_{\omega_{1} \omega}(Q)$-sentence in which $A$ and $b$ do not appear; the statement is a conjunction over sets $A$ of disjunctions over extensions of $b$.

The information about tuples of natural numbers encoded in a quantifier $Q$ of this kind is definable in $\mathscr{L}_{\omega_{1} \omega}(Q)$. This suggests the next definition.

Definition 11. A Borel quantifier $Q$ is $\operatorname{good}$ if it is monotone upwards, $\operatorname{Aut}(Q)$ is closed, and any finite injection $p: n \rightarrow \mathbb{N}$ strongly compatible with $Q$ extends to some $f \in \operatorname{Aut}(Q)$.

Examples for good quantifiers are $\forall, \exists$, "for infinitely many", "for all but finitely many", "for some prime number", "for all even numbers or for all odd numbers", and any positive boolean combination of quantifiers $Q_{A}$ as in the next section. We do not know if every monotone upwards closed quantifier is good. We also do not know whether the Lopez-Escobar theorem is true for $\mathscr{L}_{\omega_{1} \omega}\left(Q_{0}, Q_{1}\right)$ if $Q_{0}$ and $Q_{1}$ are good quantifiers.

Note that the automorphism group of a closed quantifier is closed. Conversely we have

Proposition 12. Suppose $G$ is a closed subgroup of $\operatorname{Sym}(\mathbb{N})$. There is a good binary quantifier $Q$ with $G=\operatorname{Aut}(Q)$.

Proof. Let $P$ be the downward closure of

$$
\bigcup_{k \in \mathbb{N}} \operatorname{Orb}(\{\langle 0,0\rangle,\langle 0,1\rangle,\langle 1,2\rangle, \ldots,\langle k-1, k\rangle\}) .
$$

Then $P$ is $G$-invariant, so its closure $R$ is $G$-invariant as well. Let $Q$ be the set of $A \subseteq \mathbb{N}^{2}$ with $\mathbb{N}^{2}-A \in R$. Then $Q$ is monotone upwards and $\operatorname{Aut}(Q)$ is closed. If $p: k \rightarrow \mathbb{N}$ is an injection compatible with $Q$, then

$$
s=\{\langle p(0), p(0)\rangle,\langle p(0), p(1)\rangle, \ldots,\langle p(k-2), p(k-1)\rangle\} \in R .
$$

Let $\left\langle a^{n}: n<\omega\right\rangle$ be a sequence in $P$ converging to $s$. Then $a^{n}$ eventually contains a set of the form $\left\{\left\langle a_{0}^{n}, a_{0}^{n}\right\rangle, \ldots,\left\langle a_{k-2}^{n}, a_{k-1}^{n}\right\rangle\right\}$ and the eventual value of $a_{i}^{n}$ is $p(i)$ for all $i<k$. Hence $s \in P$ and $p$ can be extended to a permutation in $G$. To show that $\operatorname{Aut}(Q)=G$, suppose $g \in \operatorname{Aut}(Q)$ and thus $\{\langle g(0), g(0)\rangle,\langle g(0), g(1)\rangle, \ldots,\langle g(m-$ $1), g(m)\rangle\} \in R$ for every $m$. This is in fact an element of $P$ by the previous 
argument, so there are permutations $h_{m} \in G$ for each $m$ with $g(i)=h_{m}(i)$ for all $i \leq m$. Since $h_{m} \rightarrow g$ we have $g \in G$.

Hence there is a correspondence between the closed subgroups of $\operatorname{Sym}(\mathbb{N})$ and good binary quantifiers $Q$. Let $\operatorname{Inv}(G)$ denote the family of closed $G$-invariant subsets of $2^{\mathbb{N}^{2}}$. Then

$$
\operatorname{Aut}(\operatorname{Inv}(G))=G
$$

for every closed subgroup $G \leq \operatorname{Sym}(\mathbb{N})$.

\section{More QUANTIFIERS}

We consider more quantifiers whose automorphism group is closed and whose orbits are definable from the quantifier, so that a version of the Lopez-Escobar theorem holds. To see that this class is neither closed under intersection nor under union, consider

Definition 13. A principal quantifier is of the form

$$
\begin{aligned}
& \text { - } Q_{A}=\left\{X \subseteq \mathbb{N}^{k}: A \subseteq X\right\} \text { or } \\
& \text { - } Q^{A}=\left\{X \subseteq \mathbb{N}^{k}: X \subseteq A\right\} \text {, }
\end{aligned}
$$

where $A$ is a subset of $\mathbb{N}^{k}$.

The automorphism group $\operatorname{Aut}\left(Q_{A}\right)=\operatorname{Aut}\left(Q^{A}\right)=\operatorname{Aut}\left(Q_{A} \cap Q^{A}\right)=\operatorname{Aut}(A)$ of a principal quantifier is closed, and its orbits are definable in $\mathscr{L}_{\omega \omega}\left(Q_{A}\right)$. For $A \subseteq \mathbb{N}$ this is true since $m \in A$ if and only if $\neg Q_{A} x(m \neq x)$ holds, and for $A \subseteq \mathbb{N}^{k}$ this is shown in subsection 3.2. Let us fix some infinite and co-infinite set $A \subseteq \mathbb{N}$ and let $Q=\{A\}$, so $\operatorname{Aut}(Q)=\operatorname{Aut}(A)$. All sentences $Q x \varphi(x, a)$ for $\mathscr{L}_{\omega_{1} \omega}$-formulas $\varphi$ and $a \in \mathbb{N}^{<\omega}$ are false, since the set $\{n: \varphi(n, a)\}$ is invariant under any permutation fixing $a$. Thus any subset of $\mathbb{N}$ defined by an $\mathscr{L}_{\omega_{1} \omega}(Q)$-formula with parameters in $m$ is either a subset of $m$ or includes $\mathbb{N}-m$. Hence the orbits of $\operatorname{Aut}(Q)$ are not definable in $\mathscr{L}_{\omega_{1} \omega}(Q)$.

3.1. Clopen quantifiers. The automorphism group of a closed quantifier is closed. To see that the orbits of a clopen quantifier $Q$ are definable from $Q$, we define its support.

Definition 14. A set $S \subseteq \mathbb{N}^{k}$ supports a quantifier $Q$ of type $\langle k\rangle$ if for all $A, B \subseteq \mathbb{N}^{k}$ with $A \cap S=B \cap S, A \in Q$ if and only if $B \in Q$. A minimal set $S \subseteq \mathbb{N}^{k}$ supporting $Q$ is called a support of $Q$.

If $Q$ has a support, then this is unique, since easily the set of $S \subseteq \mathbb{N}^{k}$ with support $Q$ is closed under finite intersections. Note that for each $a \in \mathbb{N}^{k}$, the set $\mathbb{N}^{k}-\{a\}$ supports the set of finite subsets of $\mathbb{N}^{k}$, so this does not have a support. However we have

Lemma 15. Every closed quantifier $Q$ has a support.

Proof. Suppose $S=\bigcap_{n \in \mathbb{N}} S_{n}$, where $S_{n}$ supports $Q$ for each $n \in \mathbb{N}$ and $S_{n} \subseteq S_{m}$ for $m \leq n$. If $A \in Q$ and $A \cap S=B \cap S$, then $A_{n}=\left(A \cap S_{n}\right) \cup\left(B-S_{n}\right) \in Q$ for each $n$, since $A \in Q$. Since $B$ is the limit of the sets $A_{n}$ and $Q$ is closed, $B \in Q$. Suppose $Q$ is of type $\langle k\rangle$ and $\mathbb{N}^{k}=\left\{a_{n}: n \in \mathbb{N}\right\}$. The support of $Q$ is the intersection of the sets $A_{n}$, where $A_{0}=\mathbb{N}^{k}$ and $A_{n+1}=A_{n}-\left\{a_{n}\right\}$ if this set supports $Q$ and $A_{n+1}=A_{n}$ otherwise. 
Lemma 16. The support of any clopen quantifier $Q$ is definable in $\mathscr{L}_{\omega \omega}(Q)$.

Proof. Note that a quantifier is clopen if and only if it has finite support. Suppose $Q$ is of type $\langle k\rangle$ and its support is contained in $\{0, \ldots, n-1\}^{k}$. We define $R_{l, m}(\bar{a} \frown \bar{b})$ to hold for tuples $\bar{a} \in\left(\mathbb{N}^{k}\right)^{l}$ and $\bar{b} \in\left(\mathbb{N}^{k}\right)^{m}$ if the finite partial function mapping each $a_{i}$ to 1 and each $b_{j}$ to 0 can be extended to the characteristic function of an element of $Q$. This can be easily expressed in $\mathscr{L}_{\omega \omega}(Q)$ as the existence of a tuple $\bar{c} \in\left(\mathbb{N}^{k}\right)^{n^{k}}$ so that the components of $\bar{a} \frown \bar{b} \frown \bar{c}$ are pairwise distinct and

$$
Q x\left(\bigwedge_{j<m} x \neq b_{j} \wedge\left(\bigvee_{i<l} x=a_{i} \vee \bigvee_{h<n^{k}} x=c_{h}\right)\right)
$$

holds. Then $d \in \mathbb{N}^{k}$ is in the support of $Q$ if and only if there are tuples $\bar{a} \in\left(\mathbb{N}^{k}\right)^{l}$ and $\bar{b} \in\left(\mathbb{N}^{k}\right)^{m}$ with $l+m=n^{k}-1$ such that $R_{l+1, m}(\bar{a} \frown\langle d\rangle \frown \bar{b})$ and $R_{l, m+1}(\bar{a} \frown$ $\langle d\rangle \frown \bar{b}$ ) have different truth values.

Proposition 17. The orbits of the automorphism group of any clopen quantifier $Q$ are definable in $\mathscr{L}_{\omega \omega}(Q)$.

Proof. Suppose $Q$ is of type $\langle k\rangle$ with finite support $S$. A tuple $a=\left\langle a_{i}: i\langle n\rangle\right.$ is in the orbit of a tuple $b=\left\langle b_{i}: i<n\right\rangle$ if and only if there are extensions $\left\langle a_{i}: i<l\right\rangle$ of $a$ and $\left\langle b_{i}: i<l\right\rangle$ of $b$ whose ranges contain $S$ such that the function $p$ mapping each $a_{i}$ to $b_{i}$ is well-defined and injective and $p, p^{-1}$ preserve $S$ and $R_{j, l}$ for all $j, l$ with $j+l \leq|S|$. The preservation of $S$ is expressible in $\mathscr{L}_{\omega \omega}(Q)$ as a conjunction over $S$ and the preservation of $R_{j, l}$ as a conjunction over the subsets of $S$. If this condition holds, let $f$ be any permutation of $\mathbb{N}$ extending $p$ and let $A \subseteq \mathbb{N}^{k}$. Then $A \in Q$ if and only if $A \cap S$ can be extended to a relation in $Q$ if and only if $f(A) \cap S$ can be extended to a relation in $Q$ if and only if $f(A) \in Q$; hence $f \in \operatorname{Aut}(Q)$.

If $\mathcal{Q}=\left\langle Q_{i}: i \in \mathbb{N}\right\rangle$ is a sequence of clopen quantifiers, then for each $n$, we can express that a tuple $\left\langle a_{0}, \ldots, a_{l}\right\rangle$ is extendible to a permutation compatible with $\left\langle Q_{i}: i\langle n\rangle\right.$ as in the previous proof. The conjunction of these conditions implies that $\left\langle a_{0}, \ldots, a_{l}\right\rangle$ is in the $\operatorname{Aut}(\mathcal{Q})$-orbit of $\langle 0, \ldots, l\rangle$. Hence the proposition is true for $\operatorname{Aut}(\mathcal{Q})$ and $\mathscr{L}_{\omega_{1} \omega}(\mathcal{Q})$.

3.2. Combinations of principal quantifiers. We prove that for a finite boolean combination of principal quantifiers $Q_{A_{k}}$, the automorphism group is closed and its orbits are definable from the quantifier. Suppose $\left\langle A_{k}: k<n\right\rangle$ is a partition of $\mathbb{N}^{d}$ with $d \in \mathbb{N}$ and

$$
Q=\bigcup \bigcap_{k<n} Q_{A_{k}}^{s_{i}(k)}
$$

with $s_{i} \in\{1,-1\}^{n}$ for $i<m$, where $Q_{A_{k}}^{1}=Q_{A_{k}}$ and $Q_{A_{k}}^{-1}=\neg Q_{A_{k}}$. We can assume that $n$ is minimal with these properties. In this situation we write $Q=$ $\left\langle A_{k}, s_{i}\right\rangle=\left\langle A_{k}, s_{i}: k<n, i<m\right\rangle$. We say that a tuple $\bar{a} \in\left(\mathbb{N}^{d}\right)^{<\omega}$ occurs positively (negatively) in $Q=\left\langle A_{k}, s_{i}\right\rangle$ if there is some $i$ such that for all $k, s_{i}(k)=1$ $\left(s_{i}(k)=-1\right)$ if and only if there is $j$ with $a_{j} \in A_{k}$. A tuple $\bar{a}$ occurs negatively if and only if $\psi(\bar{a}):=Q x \bigwedge_{j}\left(x \neq a_{j}\right)$ holds.

Lemma 18. If $Q=\left\langle A_{k}, s_{i}: k<n, i<m\right\rangle$, then there is an $\mathscr{L}_{\omega \omega}(Q)$-formula $\chi$ with $\chi(a, b)$ if and only if $a, b \in A_{k}$ for some $k<n$. 
Proof. Let $\chi(a, b)$ state that $\psi(\langle a\rangle \frown \bar{c}), \psi(\langle b\rangle \frown \bar{c})$, and $\psi(\langle a, b\rangle \frown \bar{c})$ have equal truth values for all tuples $\bar{c} \in\left(\mathbb{N}^{d}\right)^{n}$. If $a, b \in A_{k}$ for some $k$, then $\chi(a, b)$ holds. Suppose $a \in A_{0}, b \in A_{1}$, and $\chi(a, b)$ holds. Suppose $Q$ is the union of the sets

$$
\begin{aligned}
& \bigcup_{t \in T}\left(\neg Q_{A_{0}} \cap \neg Q_{A_{1}} \cap \bigcap_{j \geq 2} Q_{A_{j}}^{t(j)}\right), \\
& \bigcup_{u \in U}\left(Q_{A_{0}} \cap \neg Q_{A_{1}} \cap \bigcap_{j \geq 2} Q_{A_{j}}^{u(j)}\right), \\
& \bigcup_{v \in V}\left(\neg Q_{A_{0}} \cap Q_{A_{1}} \cap \bigcap_{j \geq 2} Q_{A_{j}}^{v(j)}\right), \\
& \bigcup_{w \in W}\left(Q_{A_{0}} \cap Q_{A_{1}} \cap \bigcap_{j \geq 2} Q_{A_{j}}^{w(j)}\right) .
\end{aligned}
$$

We claim that $T=U=V$. To prove $T \subseteq U$, suppose $t \in T$ and pick $\bar{d}$ so that there is exactly one $d_{k} \in A_{k}$ for each $k \geq 2$ with $t(k)=1$, so that $\psi(\langle a, b\rangle \frown \bar{d})$ holds. Then $\psi(\langle b\rangle \frown \bar{d})$ holds and hence $t \in U$. The other cases are analogous. This shows that $n$ is not minimal, since

$$
\left(\neg Q_{A_{0}} \cap \neg Q_{A_{1}}\right) \cup\left(Q_{A_{0}} \cap \neg Q_{A_{1}}\right) \cup\left(\neg Q_{A_{0}} \cap Q_{A_{1}}\right)
$$

can be replaced by $\neg\left(Q_{A_{0}} \cap Q_{A_{1}}\right)=\neg Q_{A_{0} \cup A_{1}}$.

Note that the assumption that $n$ is minimal is essential here, since otherwise the proof does not even work for quantifiers of the form $Q=Q_{A} \cap Q_{B}$.

Lemma 19. If $Q=\left\langle A_{k}, s_{i}: k<n, i<m\right\rangle$, then for each $j \leq n$ there is an $\mathscr{L}_{\omega \omega}(Q)$-formula $\theta_{j}$ such that $\theta_{j}(\bar{a}, \bar{b})$ holds if and only if

- $\bar{a}$ occurs positively and has length $j$,

- $\bar{b}$ occurs negatively and has length $n-j$, and

- all elements of $\bar{a} \frown \bar{b}$ are in different $A_{k}$.

Proof. The formula $\theta_{j}$ can be expressed by $\chi$ and $\psi$. Note that if $\bar{b}$ occurs negatively, then $\bar{a}$ has to occur positively, given the remaining conditions.

Lemma 20. If $Q=\left\langle A_{k}, s_{i}: k<n, i<m\right\rangle$, then $g \in \operatorname{Aut}(Q)$ if and only if there are a permutation $p$ of $n$ and a permutation $r$ of $m$ such that $g\left(A_{k}\right)=A_{p(k)}$ for all $k<n$ and $s_{r(i)}=s_{i} \circ p^{-1}$ for all $i<m$.

Proof. If $g \in \operatorname{Aut}(Q)$, then $g$ permutes the $A_{k}$ by the previous lemma. Let $p: n \rightarrow n$ be this permutation. For each $s: n \rightarrow\{-1,1\}$, there is $i<m$ with $s=s_{i}$ if and only if there is some $j<m$ with $s \circ p=s_{j}$. Suppose $p$ and $r$ are given and $x \in \bigcap_{k<n} Q_{A_{k}}^{s_{i}(k)}$ for some $i<n$. Then $g(x) \in \bigcap_{k<n} Q_{A_{p(k)}}^{s_{i}(k)}=\bigcap_{k<n} Q_{A_{k}}^{s_{r(i)}}$.

This implies that $\operatorname{Aut}(Q)$ is closed. Suppose $g_{k} \rightarrow g \in S_{\infty}$ with $g_{k} \in \operatorname{Aut}(Q)$ for each $k<\omega$ and let $p_{k}$ be the permutation of $n$ corresponding to $g_{k}$ in the previous lemma. Then $p_{k}$ eventually takes a fixed value $p$; hence $g$ is defined according to $p$.

Given a tuple $\bar{a} \in\left(\mathbb{N}^{d}\right)^{j}$, we can find $f: j \rightarrow n$ such that there is a tuple $\bar{c} \in\left(\mathbb{N}^{d}\right)^{n}$ with

- all $c_{i}$ are in different $A_{k}$ and $\bar{c}$ is maximal with this property, and

- $a_{i}$ and $c_{f(i)}$ are in the same $A_{k}$ for each $i<j$. 
For tuples $\bar{c}$ with this property, let $M_{\bar{a}, \bar{c}}=\left\langle\mathbb{N}, \bar{a}, \bar{c},\left\langle A_{p(k)}: k\langle n\rangle\right\rangle\right.$, where $p$ is the unique permutation of $n$ such that $c_{k} \in A_{p(k)}$. Note that the Scott sentence $\varphi_{M_{\bar{a}, \bar{c}}}$ of $M_{\bar{a}, \bar{c}}$ is equivalent to a sentence in $\mathscr{L}_{\omega_{1} \omega}(Q)$ with parameters $\bar{a}$ and $\bar{c}$, since $A_{p(k)}$ is definable from $Q$ and $c_{k}$.

Proposition 21. For any finite boolean combination $Q$ of principal quantifiers of the form $Q_{A}$, the orbits of $\operatorname{Aut}(Q)$ are definable in $\mathscr{L}_{\omega_{1} \omega}(Q)$.

Proof. Let $Q=\left\langle A_{k}, s_{i}: k<n, i<m\right\rangle$. Suppose $\bar{a}$ is a tuple of length $j$ and $f: j \rightarrow n$ and $\bar{c}$ are as above. We claim that $\bar{b} \in \operatorname{Orb}(\bar{a})$ if and only if there is a tuple $\bar{d} \in \mathbb{N}^{n}$ such that

- all $d_{i}$ are in different $A_{k}$ and $\bar{d}$ is maximal with this property,

- $b_{i}$ and $d_{f(i)}$ are in the same $A_{k}$ for each $i$,

- $M_{\bar{b}, \bar{d}} \vDash \varphi_{M_{\bar{a}, \bar{c}}, \text { and }}$

- for all $I \subseteq n, Q n\left(\bigwedge_{k \in I} n \neq d_{k}\right)$ holds if and only if $I=\left\{k<n: s_{i}(k)=-1\right\}$ for some $i<n$.

Suppose these conditions hold for $\bar{b}$ and $\bar{d}$. Since $M_{\bar{b}, \bar{d}}$ models $\varphi_{M_{\bar{a}, \bar{c}}}$, there is a permutation $g: \mathbb{N} \rightarrow \mathbb{N}$ mapping $\bar{a}$ to $\bar{b}$ and $\bar{c}$ to $\bar{d}$. Let $p: n \rightarrow n$ be the permutation of the indices of $c_{i}$ induced by this map. Then $g\left(A_{k}\right)=A_{p(k)}$ for each $k<n$. The last condition implies that for every $i<m$ there is some $j<m$ such that $s_{i} \circ p=s_{j}$. Hence $g$ preserves $Q$ by the previous lemma.

In the previous proof, the condition on the Scott sentence can be replaced by the condition (expressible in $\mathscr{L}_{\omega_{1} \omega}(Q)$ ): If $c_{i} \in A_{k}$ and $d_{i} \in A_{l}$, then $A_{k}$ and $A_{l}$ have the same size. In fact, any injection $q: n \rightarrow \mathbb{N}$ compatible with $Q$ whose domain and range contain all finite $A_{k}$ and at least one element from each $A_{k}$ extends to some $f \in \operatorname{Aut}(Q)$. Hence any monotone upwards finite boolean combination of quantifiers $Q_{A_{k}}$ is good; for instance, every positive combination is good. Note that the proposition is also valid for boolean combinations of principal quantifiers $Q^{A_{k}}$, since $Q^{A} x \varphi(x)$ can be expressed as $Q_{\neg A} x \neg \varphi(x)$, and thus any monotone upwards finite boolean combination of quantifiers $Q^{A}$ is good. For example, "for some prime number" is expressible as $\neg Q^{A}$, where $A$ is the set of non-prime numbers. However, the two types of principal quantifiers cannot be mixed by the example at the beginning of section 3 .

The proof of the proposition only uses the co-infinite part of $Q$. We do not have an example of a quantifier whose elements are all co-infinite and for which the Lopez-Escobar theorem fails.

\section{Classes of uncountable structures}

Some of the previous results generalize to the logic space for structures with domain an uncountable cardinal $\kappa$. Let us always assume $\kappa^{<\kappa}=\kappa$. We briefly describe the setting. The basic open sets of $\kappa^{\kappa}$ and the logic space

$$
X_{\tau}=\prod_{R \in \tau} 2^{\kappa^{a(R)}}
$$

of structures with domain $\kappa$ for a relational signature $\tau$ of size $\leq \kappa$, where relations are allowed to have $<\kappa$ arguments, are given by partial functions of size $<\kappa$, and the $\kappa$-Borel sets are generated by unions of length $\kappa$ and complements. The symmetric group $\operatorname{Sym}(\kappa)$ has the relative topology from $\kappa^{\kappa}$. A topological space is $\kappa$-Baire if $\bigcap_{\alpha<\kappa} U_{\alpha}$ is dense for every sequence $\left\langle U_{\alpha}: \alpha<\kappa\right\rangle$ of open dense sets. 
A quantifier of type $\langle\alpha\rangle$ on $\kappa$ is defined as a subset of $2^{\kappa^{\alpha}}$ for $\alpha<\kappa$. To see that the automorphism group of a closed quantifier $Q$ is closed, suppose $g_{\beta} \in \operatorname{Aut}(Q)$ for each $\beta<\kappa$ and $g_{\beta} \rightarrow g \in \operatorname{Sym}(\kappa)$. Then $g_{\beta}(f) \rightarrow g(f)$ and $g_{\beta}^{-1}(f) \rightarrow g^{-1}(f)$ for all $f \in 2^{\kappa^{\alpha}}$, so $f \in Q$ if and only if $g(f) \in Q$ and hence $g \in \operatorname{Aut}(Q)$.

We have the following variants of Propositions 5, 12, and 17 with analogous proofs.

Proposition 22. Suppose $G \leq \operatorname{Sym}(\kappa)$ is closed and $\kappa$-Baire and $\mathcal{F}$ is the family of $G$-orbits of elements of $\kappa^{<\kappa}$. Suppose the signature $\tau$ is disjoint from the symbols for $\mathcal{F}$. Then a subset of $X_{\tau}$ is $\kappa$-Borel and $G$-invariant if and only if it is definable in $\mathscr{L}_{\kappa^{+} \kappa}(\mathcal{F})$.

Good quantifiers are defined as in section 2.2 but finite tuples are replaced by elements of $\kappa^{<\kappa}$. The Lopez-Escobar theorem holds for good quantifiers in this setting.

Proposition 23. Suppose $G \leq \operatorname{Sym}\left(\omega_{1}\right)$ is closed and $\omega_{1}$-Baire. Then there is a good binary quantifier $Q$ with $G=\operatorname{Aut}(Q)$.

Proof. Suppose $f: \omega_{1} \rightarrow \mathcal{P}(\omega)$ is injective. The proof is analogous to the proof of Proposition 12, except that $P$ is replaced by the downward closure of the union of the orbits of

$$
\{\langle 0,0\rangle\} \cup\{\langle n, n+1\rangle: n<\omega\} \cup\{\langle n, \alpha\rangle: \omega \leq \alpha<\gamma, n \in f(\alpha)\}
$$

for $\gamma<\omega_{1}$.

Moreover if $Q$ is a good quantifier on $\omega_{1}$, then a subset of $X_{\tau}$ is $\omega_{1}$-Borel and $G$-invariant if and only if it is definable in $\mathscr{L}_{\omega_{2} \omega_{1}}(Q)$.

Proposition 24. The orbits of the automorphism group of any clopen quantifier $Q$ on $\kappa$ are definable in $\mathscr{L}_{\kappa \kappa}(Q)$.

We do not know if Proposition 23 and the analogue of Proposition 21 are true for arbitrary uncountable cardinals $\kappa$ with $\kappa^{<\kappa}=\kappa$.

\section{REFERENCES}

1. Howard Becker and Alexander S. Kechris, The descriptive set theory of Polish group actions, London Mathematical Society Lecture Note Series, vol. 232, Cambridge University Press, Cambridge, 1996. MR1425877 (98d:54068)

2. Su Gao, Invariant descriptive set theory, Pure and Applied Mathematics (Boca Raton), vol. 293, CRC Press, Boca Raton, FL, 2009. MR2455198 (2011b:03001)

3. Alexander S. Kechris, Classical descriptive set theory, Graduate Texts in Mathematics, vol. 156, Springer-Verlag, New York, 1995. MR1321597 (96e:03057)

4. E. G. K. Lopez-Escobar, An interpolation theorem for denumerably long formulas, Fund. Math. 57 (1965), 253-272. MR0188059 (32:5500)

5. Robert Vaught, Invariant sets in topology and logic, Fund. Math. 82 (1974/75), 269-294, Collection of articles dedicated to Andrzej Mostowski on his sixtieth birthday, VII. MR0363912 $(51: 167)$

Department of Philosophy, Linguistics and Theory of Science, University of GothenBurg, Box 200, 40530 Göteborg, Sweden

E-mail address: fredrik.engstrom@gu.se

Mathematisches Institut, Universität Bonn, Endenicher Allee 60, 53115 Bonn, GerMANY

E-mail address: schlicht@math.uni-bonn.de 